\title{
Migration, Human Capital Formation and the Beneficial Brain Drain
}

\author{
Hypothesis: A Note
}

\author{
Mehdi Chowdhury* \\ Bournemouth University, UK
}

\begin{abstract}
The recent brain drain literature suggests that the migration of highly skilled people can be beneficial for a country as it gives incentives to form additional human capital. We criticise this claim by developing a career concerns model and proposing that the migration opportunity as an incentive mechanism is unreliable. In addition, we show that when an individual forms two types of human capital, increased migration opportunity for one type has a negative effect on the formation of the other type. The economic benefit and full policy implications of the findings were not addressed in this paper.
\end{abstract}

Key Words: Brain Drain, Incentives, Human Capital

JEL Classifications: F22

*Correspondence to: Mehdi Chowdhury, Business School, Bournemouth University 89 Holdenhurst Road, Bournemouth, BH8 8EB, UK. E-mail: mchowdhury@bournemouth.ac.uk. Tel: +44 (0)1202 961 397. The author would like to thank Bibhas Saha, Arijit Mukherjee and the seminar participants at Bournemouth University, UK and Dhaka University, Bangladesh for comments and suggestions. The author would like to thank Sue Barnes for carefully reading the draft of the paper. All the remaining errors are the author's. 


\title{
Migration, Human Capital Formation and the Beneficial Brain Drain
}

\section{Hypothesis: A Note}

\begin{abstract}
The recent brain drain literature suggests that the migration of highly skilled people can be beneficial for a country as it gives incentives to form additional human capital. We criticise this claim by developing a career concerns model and proposing that the migration opportunity as an incentive mechanism is unreliable. In addition, we show that when an individual forms two types of human capital, increased migration opportunity for one type has a negative effect on the formation of the other type. The economic benefit and full policy implications of the findings were not addressed in this paper.
\end{abstract}

Key Words: Brain Drain, Incentives, Human Capital

JEL Classifications: F22

\section{Introduction}

The recent brain drain literature suggests that the migration of highly skilled people can be beneficial for a country as it gives incentives to form additional human capital. The literature was pioneered by works such as Stark et al. (1997), Mountford (1997), Beine et al. (2001) and Stark and Wang (2002). Docquier and Rapoport (2012) provides a detailed survey of the theoretical and empirical literature surrounding this issue. 
We argue that the central proposition of the recent brain drain literature is very similar to the concept of 'career concerns' as analysed in Holmstörm (1999), Prendergast (1999) and Bolton and Dewatripont (2004). 'Career concerns’ refers to the idea that the incentives to exert higher effort in work may come from the market. For example, when an individual works for two periods, the wage of the individual in the second period depends on the output of first period as the market regards the output of the first period as a signal of the individual's intrinsic unobservable ability. Therefore the market provides a mechanism where an individual would exert high effort in work without the presence of an incentive compatible contract. In the second period however, without any future market, the individual exerts less than the optimum effort level. The incentive to form additional human capital in the recent brain drain literature operates the same way. The incentive there comes from the international labour market, as a high return of human capital from migration induces the individual to form additional human capital. We therefore forwarded our argument by proposing a model, in a similar way as in the career concerns literature. In our model the individual forms human capital in two consecutive periods, whereas migration opportunity ceases to exist in the second period. The incentive provided by migration is only available in the first period.

In addition, we suggest that the migration opportunity can distort the formation of skills when skills are heterogeneous. By heterogeneity we mean the heterogeneity of skill types (such as Mathematics, Language) as opposed to heterogeneity of abilities (high or low as in Stark and Wang, 2002). The migration opportunity may provide incentive to only one type of skill. This has a similarity with the idea of an individual performing multiple tasks (Holmstörm and Milgrom, 1991) Though the idea of multiple skills, in relation to migration, was raised in the past 
(Todaro, 1996 cited in Docquier and Rapoport, 2012), only recently this has started to receive attention of the theoretical brain drain literature, such as Corrado and Stryszowski (2009), Stark and Zakhrenko (2012). Our paper contributes to this literature by proposing a multitask type model with different skills and shows that the migration opportunity for one skill negatively affects the optimal formation of another type of skill. The paper therefore, to some extent, is against the beneficial brain drain hypothesis. It should also be noted that the paper is itself not a work on the 'theory of incentives'. We only utilise the structures developed by the theory as such, international migration can be viewed from that perspective. It is one of the contributions of this short paper, in addition to our regular analysis of the brain drain issue.

The rest of the paper is as follows. The second section provides the basic assumptions and analyses a model with a single type of human capital. In the third section we analyse a model with two types of human capital. The forth or final section concludes the paper.

\section{One Type of Human Capital}

We assume that the representative risk neutral individual exerts effort to form human capital in two periods. There exists one type of human capital, we denote it by $h$. $\theta$ denotes the effort level. The formation of human capital as a function of $\theta$ is,

$$
h=\alpha \ln (\theta+1)+\beta \ln (\bar{\theta}+1)
$$


Where $\bar{\theta}$ is economy wide average effort, $\alpha, \beta>0$ are coefficients. Assume that the utility function is $u(x)=x$ and $c$ is the psychological disutility of making additional effort. For simplicity assume that human capital $h$ gives return equal to $h$. The net utility of the individual in term of effort is,

$$
u(\theta)=\alpha \ln (\theta+1)+\beta \ln (\bar{\theta}+1)-c \theta
$$

The equations (1) and (2) are similar to the equations used in Stark and Wang (2002) but our interpretation is different. In Stark and Wang (2002) the term $\theta$ stands for the level of human capital, $c$ is the cost of per unit human capital and $\bar{\theta}$ is the economy wide average human capital. We instead define $\theta$ as the effort, $c$ as the disutility of effort and $\bar{\theta}$ as the economy wide average effort. This interpretation provides a clearer picture of the process of formation of human capital. As our equations are almost the same as Stark and Wang (2002) it also shows that a slight modification of the original interpretation can produce a drastically different result.

Stark and Wang (2002) focused on the positive externality of human capital, captured by $\bar{\theta}$, which potential migrants do not internalise. The externality is not the focus of our analysis hence $\beta$ is considered equal to zero. Now differentiating equation (2) with respect to $\theta$ and assuming interior solution,

$$
\frac{\partial u}{\partial \theta}=\frac{\alpha}{\theta+1}-c=0
$$

$$
\text { Or, } \theta=\frac{\alpha}{c}-1
$$


Following the brain drain literature, assume that there exists an exogenous migration probability $p$. Assume that the migrants get, $\pi$ where $\pi>1$, that is migration gives higher return to human capital. The expected utility with migration is therefore,

$p u(\pi h)+(1-p) u(h)=p \pi \alpha \ln (\theta+1)+(1-p) \alpha \ln (\theta+1)=(p \pi+1-p) \alpha \ln (\theta+1)$

The net expected utility with migration is,

$u_{m}(\theta)=(p \pi+1-p) \alpha \ln (\theta+1)-c \theta$

Differentiating equation (5) with respect to $\theta$ and assuming interior solution,

$\theta=\frac{\alpha(p \pi+1-p)}{c}-1$

As $p \pi+1-p>1$, the optimal effort to form human capital and the optimal level of human capital are higher.

We can now incorporate externality in the model and show that the opportunity of migration can act as a solution to the externality problem as shown in Stark and Wang (2002). We omit the analysis as it is now merely a routine calculation. In our context what is important is the optimal effort of the individual in the second stage of the lifespan. The optimal effort of an individual increases in the first period. However if we assume that the migration opportunity is not available in 
the second period then without the presence of migration opportunity the effort of the individual drops in that period ${ }^{1}$.

The simple model we propose is capable of providing guidelines in actual issues pertaining to the formation of human capital. For example, the research outputs of many universities of developing countries are very poor though they do not lack the required expertise (e.g. PhDs from world's best universities). It is sometimes said the faculty members of these universities are not motivated enough to undertake further research after completion of their PhDs. What is often not considered is the role of incentives on individuals' research activities. The faculty members obtain $\mathrm{PhD}$ degrees to improve their CVs, to secure better positions and/or to migrate to better universities in the West. With full, partial or no realisation of these objectives, the need to acquire further human capital falls. Hence emphasis should to be placed on the continuity of incentives, instead of reliance on a market that is beyond any control.

\section{Two Types of Human Capital}

In the previous section we developed a model where an individual exerts effort to form only one type of human capital. In reality the individuals often exert effort to form different types of human capital (e.g. Mathematics and Music). The multidimensionality of effort to perform multiple tasks has been widely analysed in the literature following the seminal work of Holmström and Milgrom (1991). The

\footnotetext{
${ }^{1}$ We do not provide the calculations for the second period as it is also quite straight forward. It should be also noted that the human capital acquired in the first period may ease the process of acquiring human capital in the second period, resulting in a reduced effort for the same level of human capital. We assume no such reduction of effort in the second period which is obviously an overly simplified assumption. Together total human capital of two periods is higher, but here we emphasis on the lack of migration motivated effort in the second period.
} 
migration literature however has not looked at the multi-dimensionality of effort to form multiple human capital. The first section of the paper connected human capital with individuals' effort level. This section connects different types of efforts with different types of human capital.

In particular, we developed a model with two types of efforts and two types of human capital. In relation to the brain drain literature, we are interested in the analysis of the effects of increased migration probability of one particular human capital on the formation the other human capital. To do so we utilise a simplified version of multitask model similar to the one used in Bolton and Dewatripont (2004, page 220).

Denote human capital by $h_{i}$ where $i=1,2$. The individual exerts effort $\theta_{i}$ to form human capital $h_{i}$. Assume for simplicity that skill $h_{i}$ gives a return equal to $h_{i}$ in migrant's home. There exists a migration probability of $p_{i}$ for skill $h_{i}$. If successful then migration gives return $\pi_{i} h_{i}$ for skill $i$. Assume also that the relationship between effort and human capital is given by similar equations as was in (1). With the presence of migration probabilities, the expected return of the individual is,

$$
\begin{aligned}
& p_{1} \pi_{1} h_{1}+p_{2} \pi_{2} h_{2}+\left(1-p_{1}-p_{2}\right)\left(h_{1}+h_{2}\right) \\
& =p_{1} \pi_{1} \alpha_{1} \ln \left(\theta_{1}+1\right)+p_{2} \pi_{2} \alpha_{2} \ln \left(\theta_{2}+1\right)+\left(1-p_{1}-p_{2}\right)\left(\alpha_{1} \ln \left(\theta_{1}+1\right)+\alpha_{2} \ln \left(\theta_{2}+1\right)\right)
\end{aligned}
$$

Assume that $\pi_{i} h_{i}>\sum_{i=1,2} h_{i}$ so that migration gives a higher return just from a single skill. We ensure it by assuming that $\pi_{i}$ is sufficiently large.

Let $\psi\left(\theta_{1}, \theta_{2}\right)=\frac{1}{2}\left(c_{1} \theta_{1}^{2}+c_{2} \theta_{2}^{2}\right)+\delta \theta_{1} \theta_{2}$ be the cost or disutility of effort. If $\delta=0$, the efforts are independent. Assume $\delta>0$. The net utility in term of effort is, 


$$
\begin{aligned}
& u\left(\theta_{1}, \theta_{2}\right)=p_{1} \pi_{1} \alpha_{1} \ln \left(\theta_{1}+1\right)+p_{2} \pi_{2} \alpha_{2} \ln \left(\theta_{2}+1\right)+\left(1-p_{1}-p_{2}\right)\left(\alpha_{1} \ln \left(\theta_{1}+1\right)+\alpha_{2} \ln \left(\theta_{2}+1\right)\right) \\
& -\frac{1}{2}\left(c_{1} \theta_{1}^{2}+c_{2} \theta_{2}^{2}\right)-\delta \theta_{1} \theta_{2}
\end{aligned}
$$

Differentiating with respect to $\theta_{1}$ and assuming interior solution we get,

$$
\frac{\partial u}{\partial \theta_{1}}=\frac{p_{1} \pi_{1} \alpha_{1}}{\theta_{1}+1}+\frac{\left(1-p_{1}-p_{2}\right) \alpha_{1}}{\theta_{1}+1}-c_{1} \theta_{1}-\delta \theta_{2}=0
$$

$$
\text { We also have } \frac{\partial^{2} u}{\partial \theta_{1}^{2}}=-\frac{p_{1} \pi_{1} \alpha_{1}}{\left(\theta_{1}+1\right)^{2}}-\frac{\left(1-p_{1}-p_{2}\right) \alpha_{1}}{\left(\theta_{1}+1\right)^{2}}-c_{1} \text { and } \frac{\partial^{2} u}{\partial \theta_{1} \partial \theta_{2}}=-\delta \text {. }
$$

Assume $\left|c_{i}\right|>|\delta|$ so that the marginal cost of an effort type responses more to the change of that effort type than that of the other effort type. It ensures $\left|\frac{\partial^{2} u}{\partial \theta_{i}^{2}}\right|>\left|\frac{\partial^{2} u}{\partial \theta_{i} \partial \theta_{j}}\right|$ as such the own second order partial derivative is higher in absolute value than the cross second order partial derivative. From (8) we obtain,

$p_{1} \pi_{1} \alpha_{1}+\left(1-p_{1}-p_{2}\right) \alpha_{1}-c_{1} \theta_{1}^{2}-c_{1} \theta_{1}-\delta \theta_{1} \theta_{2}-\delta \theta_{2}=0$

By differentiating (7) with respect to $\theta_{2}$,

$$
p_{2} \pi_{2} \alpha_{2}+\left(1-p_{1}-p_{2}\right) \alpha_{2}-c_{2} \theta_{2}^{2}-c_{2} \theta_{2}-\delta \theta_{1} \theta_{2}-\delta \theta_{1}=0
$$


Differentiating totally and assuming $\pi_{i}, \alpha_{i}, c_{i}$ and $p_{2}$ unchanged, we obtain from equations (9) and (10),

$\pi_{1} \alpha_{1} d p_{1}-\alpha_{1} d p_{1}-2 c_{1} \theta_{1} d \theta_{1}-c_{1} d \theta_{1}-\delta \theta_{2} d \theta_{1}-\delta \theta_{1} d \theta_{2}-\delta d \theta_{2}=0$

and,

$-\alpha_{2} d p_{1}-2 c_{2} \theta_{2} d \theta_{2}-c_{2} d \theta_{2}-\delta \theta_{2} d \theta_{1}-\delta \theta_{1} d \theta_{2}-\delta d \theta_{1}=0$

From equations (11) and (12) we obtain,

$\frac{d \theta_{1}}{d p_{1}}=\frac{2 \pi_{1} \alpha_{1} c_{2} \theta_{2}-2 \alpha_{1} c_{2} \theta_{2}+\pi_{1} \alpha_{1} c_{2}-\alpha_{1} c_{2}+\pi_{1} \alpha_{1} \delta \theta_{1}-\alpha_{1} \delta \theta_{1}+\alpha_{2} \delta \theta_{1}+\delta \alpha_{2}}{D}$

where

$D$

$=4 c_{1} c_{2} \theta_{1} \theta_{2}+2 c_{1} c_{2} \theta_{2}+2 c_{2} \delta \theta_{2}^{2}+2 c_{1} c_{2} \theta_{1}+c_{1} c_{2}+\delta c_{2} \theta_{2}+2 c_{1} \delta \theta_{1}^{2}+c_{1} \delta \theta_{1}-\delta^{2} \theta_{1}-\delta^{2} \theta_{2}-\delta^{2}>0$ as required for the stability.

Also we have,

$2 \pi_{1} \alpha_{1} c_{2} \theta_{2}-2 \alpha_{1} c_{2} \theta_{2}+\pi_{1} \alpha_{1} c_{2}-\alpha_{1} c_{2}+\pi_{1} \alpha_{1} \delta \theta_{1}-\alpha_{1} \delta \theta_{1}+\alpha_{2} \delta \theta_{1}+\delta \alpha_{2}$ positive by

assumption. Therefore, $\frac{d \theta_{1}}{d p_{1}}>0$. Hence, when efforts are substitutes ${ }^{2}$, the effort for a

type of human capital increases as migration opportunity of that type increases.

Similarly we obtain,

${ }^{2}$ Substitutability is however not essential to obtain a similar result (see Appendix). 


$$
\frac{d \theta_{2}}{d p_{1}}=\frac{-2 c_{1} \theta_{1} \alpha_{2}-c_{1} \alpha_{2}-\delta \theta_{2} \alpha_{2}-\delta \theta_{2} \pi_{1} \alpha_{1}+\delta \theta_{2} \alpha_{1}-\delta \pi_{1} \alpha_{1}+\delta \alpha_{1}}{D}<0
$$

Therefore the effort and consequently the human capital of one type falls as the migration opportunity of the other type rises.

The result we obtained was not surprising. It was also not difficult to calculate. However, the strength of the analysis lies in its ability to clearly illustrate the effects of the higher migration probability of one type of human capital on the optimal formation of different types of human capital. As the migration probability of one type increases, the formation of that human capital increases and the formation of the other human capital decreases. The results hence provide an insight on the asymmetric nature of the effects of migration on human capital formation.

Assume further that the economy faces an external shock that increases the migration opportunity in the first period. With the higher migration opportunity, the individual increases the effort in forming $h_{1}$ and reduces the effort in forming the other capital $h_{2}$. However, the individuals' efforts return to the pre-shock levels in the second period. Therefore, if the migration opportunity distorts the formation of human capital in the first period, an absence of that in the second period helps to confront the distortion.

The effect of a shock, however, can be permanent. As discussed in footnote 1 , if the second period's human capital to some extent depends on the first period's human capital, the additional human capital of one type in one period may result in an increased formation of that type and a decreased formation of the other type in the second period. Hence an increased migration opportunity may have a lasting effect on the overall formation of human capital of an economy. 
We might conclude that the migration motivated additional human capital is a brain waste. From our analysis of the paper, however, we cannot yet make such a conclusion. The human capital is a brain waste when it is not useful for the country of emigration. To analyse it, we require a better understanding of a country's objectives and the connection between the human capital and a country's objectives. We have not defined any such objective and/or a model that is capable of analysing the welfare implications of migration. Instead we restricted the analysis in showing a weakness of recent brain drain literature that does not pay attention to the distortive influence of migration in the composition of different types of human capital within a country.

\section{Conclusion}

In this paper we suggested that international migration, when beyond the control of a country, is rather an unreliable incentive mechanism to support the formation of human capital. The individuals in different periods of lifespan face different migration opportunities. Migration opportunities may give incentives to form additional human capital in one period and none in other periods. In addition when skills are heterogeneous, the migration opportunity may distort the composition of human capital formation. We have not analysed how this distortion influences the overall economy but this paper suggests for a reconsideration of the idea of the beneficial ‘brain drain’.

The analysis of the paper is also directing towards some interesting future researches on multiple human capital. A natural extension of the analysis in this paper would be to design an incentive mechanism that is capable of the offsetting the 
negative effects, if any, of migration opportunities. Another possible way is to consider how it shapes the international trade of a country by encouraging the production of import substituting skills. We may also want to study the effects of a temporary migration opportunity on the long run formation of human capital. In future, attempts to be made for extension of the analysis in these directions. 


\section{References:}

Beine, M., Docquier, F. and Rapoport, H. (2001). 'Brain Drain and Economic Growth: Theory and Evidence', Journal of Development Economics, Vol. 64, No. 1, pp. 275-89.

Bolton, P. and Dewatripont, M. (2004). Contract Theory. MIT Press.

Corrado, D. M. and Stryszowski, P. (2009). 'Migration, Human Capital Accumulation and Development', Journal of Development Economics, Vol. 90, No. 2, pp. $306-13$

Docquier, F. and Rapoport, H. (2012). 'Globalization, Brain Drain and Development', Journal of Economic Literature, Vol. 50, No. 3, pp. 681-730.

Mountford, A. (1997). 'Can a Brain Drain be Good for Growth in the Source Economy?’, Journal of Development Economics, Vol. 53, No. 2, pp. 287-03.

Holmström, B. (1999). 'Managerial Incentive Problems: A Dynamic Perspective', Review of Economic Studies, Vol. 66, No. 1, pp. 169-182.

Holmström, B. and Milgrom, P. (1991). 'Multitask Principal-Agent Analysis: Incentive Contracts, Assets Ownership, and Job Design', Journal of Law, Economics and Organization, Vol. 7, pp. 24-52.

Prendergast, C. (1997). 'The Provision of Incentives in Firms, Journal of Economic Literature', Vol. 37, No. 1, pp. 7-63

Stark, O., Helmenstein, C. and Prskawetz, A. (1997). 'A Brain Gain with Brain Drain’, Economics Letters, Vol. 55, Issue 2, pp. 227-234.

Stark, O. and Wang, Y. (2002). 'Inducing Human Capital Formation: Migration as a Substitute for Subsidies’, Journal of Public Economics, Vol. 86, No.1, pp. 29-46. 
Stark, O. and Zakharenko, R. (2012). Differential Migration Prospects, Skill Formation and Welfare, Review of International Economics, Vol. 20, No. 4, 657-673.

Todaro, M. (1996). Economic Development, 5th ed., Longman Publishing. 


\section{Appendix}

In this appendix we assume that $\delta=0$, The equations (9) and (10) respectively become,

$p_{1} \pi_{1} \alpha_{1}+\left(1-p_{1}-p_{2}\right) \alpha_{1}-c_{1} \theta_{1}^{2}-c_{1} \theta_{1}=0$

$\pi_{1} \alpha_{1} d p_{1}-\alpha_{1} d p_{1}-2 c_{1} \theta_{1} d \theta_{1}-c_{1} d \theta_{1}=0$

Therefore,

$\frac{d \theta_{1}}{d p_{1}}=\frac{\left(\pi_{1}-1\right) \alpha_{1}}{2 c_{1} \theta_{1}+c_{1}}>0$

The equations (11) and (12) respectively become,

$$
\begin{aligned}
& p_{2} \pi_{2} \alpha_{2}+\left(1-p_{1}-p_{2}\right) \alpha_{2}-c_{2} \theta_{2}^{2}-c_{2} \theta_{2}-\delta \theta_{1} \theta_{2}-\delta \theta_{1}=0 \\
& -\alpha_{2} d p_{1}-2 c_{2} \theta_{2} d \theta_{2}-c_{2} d \theta_{2}=0
\end{aligned}
$$

Implying that,

$$
\frac{d \theta_{2}}{d p_{1}}=-\frac{\alpha_{2}}{2 c_{2} \theta_{2}+c_{2}}<0
$$

Therefore substitutability is not essential to demonstrate that the increased migration opportunity of one skill has a negative effect on the formation of the other skill. 